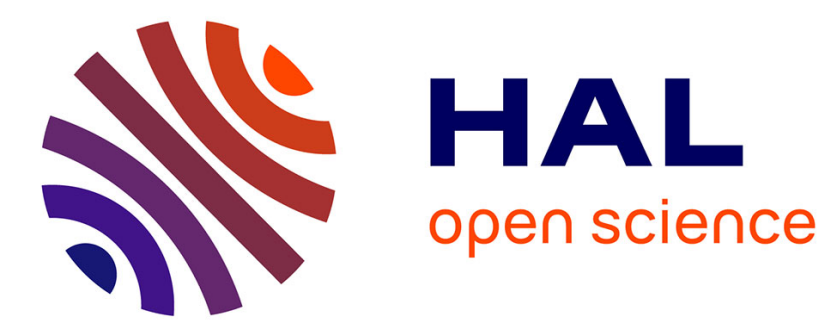

\title{
New insights into the evolutionary conservation of the sole PIKK pseudokinase Tra1/TRRAP
}

\author{
Alberto Elías-Villalobos, Philippe Fort, Dominique Helmlinger
}

\section{To cite this version:}

Alberto Elías-Villalobos, Philippe Fort, Dominique Helmlinger. New insights into the evolutionary conservation of the sole PIKK pseudokinase Tra1/TRRAP. Biochemical Society Transactions, 2019, 47 (6), pp.1597 - 1608. 10.1042/BST20180496 . hal-02394910

\section{HAL Id: hal-02394910 \\ https://hal.umontpellier.fr/hal-02394910}

Submitted on 17 Nov 2020

HAL is a multi-disciplinary open access archive for the deposit and dissemination of scientific research documents, whether they are published or not. The documents may come from teaching and research institutions in France or abroad, or from public or private research centers.
L'archive ouverte pluridisciplinaire HAL, est destinée au dépôt et à la diffusion de documents scientifiques de niveau recherche, publiés ou non, émanant des établissements d'enseignement et de recherche français ou étrangers, des laboratoires publics ou privés. 
1 New insights into the evolutionary conservation of the sole PIKK pseudokinase

2 Tra1/TRRAP

3

$4 \quad$ Alberto Elías-Villalobos ${ }^{1}$, Philippe Fort ${ }^{1}$, and Dominique Helmlinger ${ }^{1}$

5

$6{ }^{1}$ CRBM, CNRS, University of Montpellier, Montpellier, France

7

$8 \quad{ }^{*}$ Corresponding author: Dominique Helmlinger

9 Phone: $+33-434-35-95-51$

10 Fax: +33-434-35-94-10

11 Email: $\underline{\text { dhelmlinger@crbm.cnrs.fr }}$

12

Keywords: pseudokinase, chaperones, transcription, chromatin, macromolecular complex 
1

2 Phosphorylation by protein kinases is a fundamental mechanism of signal transduction. Many

\section{ABSTRACT} kinase families contain one or several members that, although evolutionarily conserved, lack the residues required for catalytic activity. Studies combining structural, biochemical, and functional approaches revealed that these pseudokinases have crucial roles in vivo and may even represent attractive targets for pharmacological intervention. Pseudokinases mediate signal transduction by a diversity of mechanisms, including allosteric regulation of their active counterparts, assembly of signaling hubs, or modulation of protein localization. One such pseudokinase, named Tra1 in yeast and TRRAP in mammals, is the only member lacking all catalytic residues within the PIKK family of kinases. PIKKs are related to the PI3K family of lipid kinases, but function as Serine/Threonine protein kinases and have pivotal roles in diverse processes such as DNA damage sensing and repair, metabolic control of cell growth, nonsense mediated decay, or transcription initiation. Tra1/TRRAP is the largest subunit of two distinct transcriptional co-activator complexes, SAGA and NuA4/TIP60, which it recruits to promoters upon transcription factor binding. Here, we review our current knowledge on the Tra1/TRRAP pseudokinase, focusing on its role as a scaffold for SAGA and NuA4/TIP60 complex assembly and recruitment to chromatin. We further discuss its evolutionary history within the PIKK family and highlight recent findings that reveal the importance of molecular chaperones in pseudokinase folding, function, and conservation. 


\section{Introduction.}

A pseudokinase is defined as a kinase paralog that lacks essential catalytic residues and is thus predicted to have no or weakened phosphotransfer activity. Similar to other pseudoenzymes, pseudokinases have been first considered as evolutionary remnants of their active counterparts. However, analysis of their phylogenetic distribution and conservation revealed their prevalence across all kingdoms of life and in many distinct kinase families. Furthermore, their evolutionary conservation outside of catalytic residues clearly argue for important functional roles [1,2]. Pseudokinases have finally received increased attention over the past two decades. Their study provided novel insights into the function of catalytically active kinases, but also revealed their specific roles in many fundamental processes.

Structural, biochemical, and genetic evidence indicate that pseudokinases perform critical non-enzymatic functions in signaling pathways. Their protein-protein interaction domains can allosterically regulate the activities of cognate kinases or other enzymes, compete for substrate binding, scaffold the assembly of signaling complexes, or modulate protein trafficking and localization. Additionally, some pseudokinases with noncanonical catalytic residues show residual activity or ATP binding, which can have a specific regulatory function (for more comprehensive reviews, see [3-8]). Adding to this diversity of mechanisms, recent work showed that the SelO pseudokinase is an active enzyme that uses ATP to transfer AMP to specific substrates [9]. It is therefore becoming clear that much remains to be learned from the study of pseudokinases, and more generally pseudoenzymes [10,11]. Finally, their functions are relevant to numerous human diseases, including cancer, and pseudoenzymes represent attractive targets for novel therapeutic strategies [12].

In this mini-review, we will focus on the structure, function, and evolutionary history of one such pseudokinase, called the transformation/transcription domain-associated protein (TRRAP) in mammals or Tra1 in yeast $[13,14]$. TRRAP is the sole inactive member of a family of atypical kinases, named phosphatidylinositol 3-kinase related kinase (PIKK), which 
comprises the catalytic subunit of the DNA-dependent protein kinase (DNA-PK), ataxiatelangiectasia mutated (ATM), ATM and Rad3-related (ATR), target of rapamycin (TOR), and suppressor of morphogenesis in genitalia 1 (SMG1).

\section{The PIKK family of protein kinases.}

PIKKs are related to the phosphatidylinositol class of lipid kinases (PI3K), but function as Serine/Threonine protein kinases, mediating signal transduction in diverse biological contexts (for a review on PIKK fucntions, see [15]). DNA-PK is a critical effector during DNA doublestrand break repair. ATM and ATR are the central components of the DNA damage checkpoint and are activated in response to various genotoxic stresses. TOR is a central regulator of metabolism, growth, and survival in response to nutrient availability, growth factors, hormones and stress signals. SMG1 mediates the decay of mRNAs with premature stop codons or that were inappropriately spliced. Finally, the only pseudokinase of this family, TRRAP, has essential roles during transcription. Functional studies in different model systems, including yeasts, nematodes, flies, and mice, have established that these distinct functions are conserved across eukaryotes.

Despite these diverse functions, PIKKs are structurally related and share a characteristic domain architecture. All PIKKs are large proteins, which size ranges from approximately 250 to $470 \mathrm{kDa}$ in humans (Figure 1A). Long arrays of $\alpha$-solenoids, termed Huntingtin, EF3A, PP2A, TOR (HEAT) repeats, precede a region with high similarity between PIKKs and formed by solenoidal TPR repeats, called the FRAP, ATM, and TRRAP domain (FAT). These extended superhelical structural motifs are immediately followed by the highly conserved, PI3K-related, kinase domain and a short C-terminal FATC motif (Figure 1A). Although the catalytic domain of PIKKs is homologous to that of PI3Ks, notable differences exist in the catalytic motifs (Figure 1B). As compared to PI3Ks, PIKKs contain only the Lys residue from the ATP-binding motif VAIK and the Asp residue from the divalent cation-binding motif DFG. Indeed, PIKKs are strict S/T protein kinases with no reported lipid substrates. 
In spite of their sequence similarity, PIKKs differ markedly in their structural organization, mostly of their $\mathrm{N}$-terminal repeats, oligomerization, and interaction with regulatory factors $[16,17]$. For example, TOR constitutively dimerizes and interacts with accessory proteins to form either TOR complex 1 (TORC1) or TOR complex 2 (TORC2), which are defined by the specific incorporation of either regulatory associated protein of MTOR complex 1 (RPTOR) or RPTOR independent companion of MTOR complex 2 (RICTOR), respectively. In contrast, DNA-PK and ATM dimerization is regulatable and activates DNA-PK, whereas it inhibits ATM activity. Finally, the 'pseudoPIKK' TRRAP is always monomeric but part of larger macromolecular assemblies, the Spt-Ada-Gcn5 acetyltransferase complex (called SAGA both in yeasts and mammals) and the nucleosome acetyltransferase of $\mathrm{H} 4$ complex (called NuA4 in yeasts and TIP60 in mammals).

The phylogenetic distribution of PIKKs shows variations between clades, both in the identity of PIKKs and in the number of PIKK paralogs (Figure 2A). TOR, ATM, ATR, and TRRAP are ubiquitously distributed across all major eukaryotic lineages, whereas DNA-PK and SMG1 were lost from several lineages. For example, fungal species from the dikarya lineage, which includes the model organism Saccharomyces cerevisiae, lost DNA-PK and SMG1 but have two TOR paralogs, Tor1 and Tor2. Similarly, some Taphrinomycetes have acquired two TRRAP paralogs, Tra1 and Tra2, which have non-redundant functions in the model organism Schizosaccharomyces pombe $[18,19]$. DNA-PK is also absent from the Diptera and Nematoda clades, while SMG1 appears absent from the Chlorophyta and Alveolata, which are clades of plants and protists, respectively. Finally, unicellular parasites from the Excavata clade show striking differences to these general principles, most notably the loss of several PIKKs, which is likely related to their parasitic lifestyle and was noted previously [20]. For example, we observe that, except TOR, all PIKKs were lost from Giardia intestinalis, while Trichomonas vaginalis appear to have 38 TOR paralogs, 3 TRRAP paralogs, and 2 ATR paralogs. Overall, this analysis reveals that the only 'pseudoPIKK', 
1 Tra1/TRRAP, is conserved throughout all eukaryotic clades and is therefore probably an

2 ancestral member of the PIKK family with important cellular functions.

\section{The Tra1/TRRAP pseudokinase.}

Although Tra1/TRRAP is enzymatically inactive, it shows the large size and typical domain architecture of all active PIKKs (Figure 1A). Detailed analysis of Tra1/TRRAP PI3K-like kinase domain indicates that all orthologs and paralogs have lost the three motifs that are essential for enzymatic activity $[13,19]$. These include the ATP-binding motif VAIK, the catalytic motif $H R D$, and the divalent cation-binding motif DFG (Figure 1B,C). We note that residues from the catalytic motifs are not conserved across different clades, further arguing that Tra1/TRRAP does not possess enzymatic activity (Figure 1C). In addition, a phylogenetic tree of PIKKs from all major eukaryotic clades confirm that TRRAP is likely an ancestral pseudo-enzyme because its PI3K-like kinase domain diverges more than that of active PIKKs (Figure 2B). Examination of the branch points of this tree suggests that TRRAP is more closely related to DNA-PK than other PIKKs and might have thus originated from its early duplication. However, this hypothesis is not fully supported by a bootstrap analysis and statistical calculations (Figure 2B).

Two recently published studies brought unprecedented insights into the structure of Tra1 and the topological organization of its domains, in particular due to advances in cryo-electron microscopy (EM) approaches [21,22]. Both structures identified three distinct regions within the N-terminal array of HEAT repeats, named the 'Finger', 'Ring', and 'Clasp' domains, which fold into an $\alpha$-solenoid superhelical structure. The remaining FAT, kinase, and FATC domains form a globular region, termed 'Head', which is often referred to as 'FATKIN' in other PIKKs and adopts a conformation that is highly conserved between them $[17,23]$. In contrast, the topology of the HEAT domain is much more variable between PIKKs. Interestingly, the Tra1 HEAT domain adopts a 'diamond ring' conformation, which is similar to that of DNA-PK [24], despite apparent low sequence similarity but consistent with their phylogenetic relatedness. 


\section{The Tra1/TRRAP 'pseudoPIKK': a protein interaction hub for activator targeting.}

Early work hypothesized that the function of Tra1/TRRAP depends on its extensive protein interaction surfaces rather than its pseudokinase domain, similar to a few other pseudokinases such as the Integrin-linked kinase (ILK) or the Tribbles (TRIB) pseudokinases. Although many studies confirmed the importance of protein-protein interaction in Tra1/TRRAP function, mutational analyses of $S$. cerevisiae Tra1 indicated that its pseudokinase domain has also critical roles in vivo [25-27]. Similarly, recent work established that the atypical sequence of the TRIB pseudokinase domain relates to an important functional fold, whose plasticity modulates substrate ubiquitination or assembly of signaling modules [28-30].

TRRAP was originally identified as an interacting partner of the c-MYC and E2F transcription factors and is essential for their oncogenic activities during transformation [13]. Since this discovery, numerous biochemical and genetic studies in different organisms established that a diverse range of activators require Tra1/TRRAP to initiate transcription [26,31-38]. Several elegant studies have demonstrated in vivo, physical interaction between Tra1 and the transactivation domain of activators [39-44]. Mapping the regions of Tra1 that are involved suggests that its HEAT domain is the main interaction surface for several transcription factors, including Gal4, Gcn4, and Rap1 in S. cerevisiae, or c-MYC and p53 in mammalian cells. Indeed, the HEAT domain forms a large exposed surface on Tra1, even when integrated within the SAGA or NuA4 complexes $[22,45]$. However, the molecular details by which activators interact with Tra1 remains elusive and might involve dynamic, low specificity mechanisms, analogous to Gcn4-Mediator interaction [46]. In addition, whether distinct activators can simultaneously bind to one Tra1/TRRAP molecule remains to be demonstrated, but is conceivable because activators selectively target distinct, nonoverlapping regions distributed across the HEAT domain $[26,38]$.

\section{Scaffolding role of the Tra1/TRRAP 'pseudoPIKK' within transcription complexes.}


1 Tra1/TRRAP is predominantly found within either one of two distinct co-activator complexes,

2 SAGA and NuA4/TIP60 [14,47-49]. Both co-activators are highly conserved regulators of

3 transcription initiation and are large multimeric complexes with modular organization (for reviews on SAGA and NuA4/TIP60, see [50-52]). SAGA carries histone H3 acetylation (HAT) and histone H2B de-ubiquitylation (DUB) activities, and modulates the recruitment of the TATA-box binding protein (TBP) to core promoters. Yeast NuA4 acetylates histone $\mathrm{H} 4$, $\mathrm{H} 2 \mathrm{~A}$, and the histone variant $\mathrm{H} 2 \mathrm{~A} . \mathrm{Z}$, while the mammalian homologous complex, TIP60, also contains an ATPase subunit, P400, which catalyzes H2A.Z deposition. Altogether, Tra1/TRRAP large size, lack of catalytic activity, and ability to interact with many transcription factors suggested that its primary function during transcription is to scaffold and recruit these complexes to specific promoters.

Recent work from our group have clarified this model, at least in fission yeast. Indeed, $S$. pombe provides a unique opportunity to study Tra1/TRRAP function because, in marked contrast with $S$. cerevisiae and mice, a tra1 $\Delta$ deletion mutant is viable in $S$. pombe [53]. We demonstrated that Tra1 is not required for viability in $S$. pombe because its genome has two paralogous genes, tra1+ and tra2+, and each has non-redundant roles that are specific for SAGA or NuA4, respectively [19,54]. Phylogenetic analyses indicate that these paralogs result from a duplication of a single gene in the ancestor of the Schizosaccharomyces lineage, suggesting that each paralog diverged such that Tra1 and Tra2 are specific for each complex in S. pombe [19]. Recently, we observed that the conditional loss of Tra2 disrupts NuA4 integrity and affects cell viability, indicating that Tra2 does indeed scaffold the assembly of the entire NuA4 complex [55]. In marked contrast, we showed that Tra1 has no scaffolding function within SAGA, but, rather, regulates the expression of a small subset of SAGA-dependent genes and specifically controls the incorporation of the DUB module [55]. Therefore, contrary to its general scaffolding role in NuA4 assembly, Tra1 has specific regulatory roles in SAGA structural organization and activity. 
These results are consistent with recent structural studies using cryo-EM and crosslinking coupled to mass spectrometry (CXMS) analyses [22,45,56-58]. Indeed, Tra1 is localized at the periphery of SAGA, which contacts the FAT domain through a surprisingly small and flexible region called the 'Hinge'. We recently demonstrated that Spt20 is the major interacting partner of Tra1 within SAGA, in both S. pombe and S. cerevisiae [55]. In contrast, Tra1 occupies a more central position within NuA4, which subunits make extensive contacts with the FAT, kinase, and FATC domains, explaining the essential role of Tra1 in NuA4 complex integrity and probably the sensitivity of these domains to mutations in vivo $[26,27,59,60]$.

To conclude, integrating structural, biochemical, and functional approaches clearly established that Tra1/TRRAP is a pseudoenzyme acting as a protein-protein interaction hub. Despite having no 'writing' activity, Tra1/TRRAP can 'read' cues from promoter-bound transcription factors and relay this signal by recruiting and/or assembling co-activator complexes. Their activities then modify chromatin and stimulate pre-initiation complex assembly to elicit specific transcriptional responses. To date, little is known about the molecular mechanisms by which Tra1/TRRAP controls SAGA and NuA4/TIP60 activities at specific promoters but these recent results have undoubtedly opened new perspectives.

\section{A specific chaperone machinery links Tra1/TRRAP to active PIKKs.}

Despite these advances in our understanding of the functional roles of this pseudokinase, the reason for the evolutionary conservation of a typical PIKK domain architecture in Tra1/TRRAP remained a mystery for years. Work from several laboratories recently provided an unexpected and elegant explanation.

Seminal work from Titia de Lange's group first reported a role for a protein called TELO2 in the stabilization of all six mammalian PIKKs, including TRRAP [61]. TELO2 is the mammalian ortholog of $S$. cerevisiae Tel2, which was identified in the first screen for mutants for shortened telomeres [62]. Concurrently, biochemical analysis of TOR complexes in fission yeast identified a trimeric complex composed of Tel2 and two additional proteins, named Tel 
two interacting proteins 1 and 2 (Tti1 and Tti2) [18,63]. All three subunits are conserved between yeasts and mammals and form the Triple-T complex (TTT). Further studies in yeast and mammals established that TTT is a novel HSP90 cochaperone dedicated to PIKK stabilization and assembly into active complexes (Figure 3) [61,64-68]. Numerous functional studies in different organisms implicated TTT in PIKK signaling in response to DNA damage or metabolic stress $[61,64,66-72]$. Importantly, TTT interacts genetically and physically with Tra1 in yeast $[18,19,73-75]$ and stabilizes TRRAP in human cells $[61,66-68]$. Accordingly, we recently established that, in fission yeast, Hsp90 and TTT promote the de novo incorporation of Tra1 into SAGA and of Tra2 into NuA4 [55].

Altogether, these findings have two important implications. First, Tra1/TRRAP, the only 'pseudoPIKK', shares a dedicated chaperone machinery with active PIKKs for its folding and assembly into larger, multimeric complexes (Figure 3). We propose that the requirement of PIKKs for a specific cochaperone explains the selection pressure on the sequence and domain organization of Tra1/TRRAP, despite the divergence of its PI3K-like kinase domain. Supporting this possibility, analysis of the phylogenetic distribution of TELO2, TTI1, and TTI2 indicates that both TELO2 and TTI1 are ancient proteins, because orthologs were found in the genomes of species representative of all major eukaryotic clades, similar to PIKKs (Figure 2A). Interestingly, TTI2 was lost from several lineages, including Diptera and Nematoda, suggesting that its function might not be strictly essential.

Second, although PIKKs are implicated in diverse processes, they are all dependent on HSP90 and its cochaperone TTT for their maturation (Figure 3). HSP90 is indeed a pleiotropic chaperone and typically requires a cochaperone to target and fold a particular subset of substrates, named clients (reviewed in [76]). For example, many HSP90 client kinases are recognized and recruited by the CDC37 cochaperone [77]. It is possible that atypical kinase clients, such as PIKKs, require a specific factor for their recruitment and folding by HSP90. Although the exact mechanism by which HSP90 and TTT promote PIKK maturation remains unknown, we propose that their massive size, unique domain 
architecture, obligate partner interactions, and substantial structural flexibility necessitate a dedicated chaperone machinery.

We note that TTT might function as a PIKK-specific adapter, rather than a cochaperone, because its binds HSP90 indirectly. Elegant structural and biochemical studies demonstrated that TELO2 phosphorylation is essential for interaction with a complex called R2TP. This multimeric cochaperone is formed by the RuvB-like AAA+ATPases RUVBL1 and RUVBL2, the PIH1 domain containing 1 protein (PIH1D1), and the RNA polymerase II associated protein 3 (RPAP3), which TPR domain directly contacts HSP90 (for a review, see $[78,79]$ ). However, PIH1D1 and RPAP3 orthologs are absent from fission yeast (G. Lledo, B. PradetBalade, and D. Helmlinger; unpublished observations) [80], suggesting either that TTT can bind HSP90 directly in some conditions, or that other factors mediate this interaction. One such factor might be the highly conserved WD40 domain-containing protein Asa1, which copurifies with TTT in both S. cerevisiae and in S. pombe [73]. Interestingly, a recent study suggests that, in S. cerevisiae, Asa1 promotes constitutive TTT-dependent stabilization of Mec1 $1^{\text {ATR }}$ and Tel1 ${ }^{\text {ATM }}$, whereas the R2TP complex replaces Asa1 in response to stress signals [80].

\section{Conclusions}

The widespread phylogenetic distribution and high conservation of seemingly inactive enzymes argue for important catalytic-independent functions. Here, we reviewed and discussed our current knowledge on the only inactive member of the PIKK family of atypical kinases. The discovery of a PIKK-specific cochaperone illuminates the importance of folding and structure in the selective pressure exerted on the sequence of pseudokinases during evolution, and perhaps more generally on pseudoenzymes. As summarized below, several important questions are of particular interest for future research on the Tra1/TRRAP 'pseudoPIKK'. The exact roles of Tra1/TRRAP during transcription are still not fully understood and its study promises many more exciting discoveries in the near future. 


\section{Perspectives}

- Tra1/TRRAP recruitment to chromatin: Our current view is that DNA-bound transcription factors are responsible for Tra1/TRRAP recruitment to chromatin. However, amongst PIKKs, Tra1/TRRAP is phylogenetically and structurally most related to DNA-PK, which can bind DNA directly [81]. The $3.5 \mathrm{~nm}$-wide opening created by the ring-like conformation of the HEAT repeats of yeast Tra1 might accommodate such a large macromolecule [21,22].

- $\quad$ Function of the PI3K-like kinase domain: Although Tra1/TRRAP lost the ability to bind ATP, it might retain the ability of PI3Ks to bind phosphatidylinositol, recognize another phospholipid, or interact with another negatively charged metabolite. Supporting this possibility, a genetic suppressor screen revealed the functional importance of exposed, positively charged residues in the cleft region of the PI3K-like domain of $S$. cerevisiae Tra1 [27].

- Allosteric regulation of chromatin-modifying activities: pseudokinases can function as pseudoscaffolds modulating the availability of substrates to enzymes (reviewed in [82]). To date, no interaction between Tra1/TRRAP and any PIKK has been reported. Rather, we speculate that Tra1/TRRAP might control the HAT, DUB, or ATPase enzymatic activities of SAGA and NuA4/TIP60. In S. cerevisiae, specific tra1 mutants decrease SAGA or NuA4 HAT activities without affecting their integrity or recruitment $[25,26]$. In $S$. pombe, we found that Tra1 controls the interaction of the DUB module with SAGA [55]. Tra1/TRRAP might regulate these enzymatic activities by an allosteric mechanism, either through conformational changes within the complex or by controlling accessibility to nucleosomal substrates. Interestingly, although the topology of Tra1 is remarkably rigid $[21,23]$, its contact point with the rest of SAGA appears very flexible [22]. Thus, the relative position of Tra1 to the other functional modules of SAGA might be regulated and used to dictate specific regulatory roles. 


\section{FIGURE LEGENDS}

Figure 1: Domain architecture and structural features of human PIKKs.

(A) Cartoons depicting the domain architecture (top, colored annotations) and structural features (bottom) of all six PIKKs from Homo sapiens (from top to bottom): TRRAP, DNA-PK, ATM, ATR, TOR, and SMG1. Residue numbers indicate the limits of each domain, which were defined based on the most recent structures available [17] and multiple alignments.

(B,C) Multiple alignments of the VAIK, DXXXXN, and DFG catalytic motifs from all human PIKKs (B) and of the corresponding regions from selected Tra1/TRRAP homologs (C), using Clustal Omega [83]. Residues that are identical or similar to the consensus sequence are shaded in black or grey background, respectively, using Boxshade 3.2. The canonical VAIK, DXXXXN, and DFG motifs are highlighted and are clearly absent from all Tra1/TRRAP orthologs and paralogs. (B) Multiple alignment of human ATR (Q13535), ATM (Q13315), TOR (P42345), SMG1 (Q96Q15), DNA-PK (PRKDC, P78527), TRRAP (Q9Y4A5), and one PI3K kinase (PI3KC3, Q8NEB9). (C) Multiple alignment of S. pombe Tra2 (Q10064), Tra1 (Q9HFE8), S. cerevisiae Tra1 (P38811), Neurospora crassa Tra1 (Q7S7K6), Arabidopsis thaliana Tra1 (F4IPJ1), Homo sapiens TRRAP (Q9Y4A5), Mus musculus Trrap (Q80YV3), Danio rerio Trrap (A0A0R4IPE4), and Drosophila melanogaster TRRAP (Nipped-A, Q8I8U7).

\section{Figure 2: Phylogenetic distribution of PIKKs and of the TTT cochaperone.}

(A) Conservation of the TTT complex subunits TELO2, TTI1, TTI2, and of the six PIKKs, TRRAP, DNA-PK (PRKDC), TOR, SMG1, ATM, and ATR across Eukaryotes. All TTT subunits are colored in green whereas each PIKK is colored independently. Orthologs that were not found are indicated by X. Numbers in colors indicate the number of paralogs found in a specific species or lineage when more than 2 were detected. ${ }^{\text {T}}$ Two copies in Taphrinomycotina; bonly found in Selaginellaceae; ' low BLAST scores; donly in Phytophthora infestans; ${ }^{e}$ lack the DXXXXN and DFG motifs and are more closely related to the TRRAP cluster. 
1 (B) TRRAP, DNA-PK (PRKDC), TOR, SMG1, ATM, and ATR PIKK subfamilies were already

2 present in early eukaryotes. Gene models encoding PIKK kinase domains were retrieved in

3 the following taxons: V: Vertebrates (Homo sapiens, NP_000042; NP_001175; NP_008835; CAC21449; NP_055907; NP_001231509), FA: Fungi Ascomycota (Schizosaccharomyces pombe, BAA33817.1; NP_595357; NP_596275; NP_595359; NP_595777; NP_592862), FM: Fungi Mucoromycota (Bifiguratus adelaidae, OZJ03251; OZJ02458; OZJ04327; OZJ04505; OZJ06116), A: Amoebozoa (Dictyostelium discoideum, XP_640504; XP_643468; XP_640629; XP_640856; XP_635176), AT: Archaeplastida Tracheophyta (Selaginella moellendorffii, EFJ08875; EFJ10333; EFJ09668; EFJ31213; EFJ26294; EFJ26034), AC: Archaeplastida Chlorophyta (Chlamydomonas reinhardtii, XP_001693670; XP_001701957; XP_001698462; XP_001697578; PNW79003), E: Euglenozoa (Leishmania major strain Friedlin, CBZ11901; CAJ08666; CAJ08193; CAJ09256), H: Heterolobosea (Naegleria gruberi, XP_002682730; XP_002674657; XP_002674694; XP_002680550; XP_002680058; XP_002682330), P: Parabasalia (Trichomonas vaginalis, XP_001583998; XP_001324760; XP_001329568; XP_001317657), D: Diplomonanida (Giardia intestinalis, ESU36088). The tree was rooted with PI3K sequences (BAE06077, NP_594699, XP_001689631, XP_001683719, XP_636122). Note that T. vaginalis genome encodes additional PIKK sequences (2 ATR, 2 TRRAP and 36 TOR). The phylogenetic tree was deduced from multiple sequence alignment of the kinase/pseudokinase domain and processed by maximum-likelihood (PhyML) and bayesian (MrBayes) analyses. Only nodes of biological importance for PIKK clustering are indicated. Numbers in red represent PhyML bootstrap proportion (in \%) and MrBayes posterior probability.

Figure 3: A dedicated chaperone machinery promotes the maturation and assembly of all PIKK kinases.

Shown is a working model of the HSP90-R2TP-TTT chaperone machinery promoting the maturation and/or assembly of PIKKs. See text for details. Their structures are shown either alone or within their respective complexes with, from top to bottom, SMG1 (EMD-2666 [84]), 
1 TOR (PDB ID: 6BCX [85] for TORC1 complex and PDB ID: 5ZCS [86] for TORC2 complex),

2 TRRAP (EMD-3804 [22] for SAGA complex and PDB ID: 5 Y81 model from EMD-6816 [45]

3 for the partial NuA4-TEEAA subcomplex), DNA-PK (PDB ID: 5LUQ [24]), ATM closed dimer

4 conformation (PDB ID: 5NP0 [87]), ATR (PDB ID: 5X60 [88] for Mec1 ${ }^{\text {ATR }}$ in complex with

5 Ddc2 $\left.{ }^{\text {ATRIP }}\right)$. The dashed lines identify the PIKK. Images were taken either from the RCSB

6 PDB (rcsb.org) and processed using the NGL viewer [89] or directly from the EMDB

7 (emdataresource.org).

8 


\section{Acknowledgments}

2 We thank members of the Helmlinger lab for stimulating discussions. The work in our

3 laboratory reviewed herein was supported by a postdoctoral fellowship from the Fondation

4 pour la Recherche Médicale to A.E.V and by funds from the CNRS (ATIP-Avenir), the FP7

5 Marie Curie Actions (FP7-PEOPLE-2012-CIG/COACTIVATOR), and the Agence Nationale

6 de la Recherche (ANR-15-CE12-0009-01 and ANR-15-CE11-0022-03) to D.H..

\section{$7 \quad$ Author contributions}

8 P.F. performed all phylogenetic analyses. A.E.V. and D.H. wrote the manuscript and all

9 authors read and approved the manuscript.

\section{Conflicts of interest}

11 The Authors declare that there are no conflicts of interest associated with the manuscript. 


\section{REFERENCES}

[1] Manning G, Whyte DB, Martinez R, Hunter T, Sudarsanam S. (2002) The protein kinase complement of the human genome. Science. 298, 1912-1934.

[2] Kwon A, Scott S, Taujale R, Yeung W, Kochut KJ, Eyers PA, et al. (2019) Tracing the origin and evolution of pseudokinases across the tree of life. Sci. Signal. 12, eaav3810.

[3] Boudeau J, Miranda-Saavedra D, Barton GJ, Alessi DR. (2006) Emerging roles of pseudokinases. Trends Cell Biol. 16, 443-452.

[4] Zeqiraj E, van Aalten DM. (2010) Pseudokinases-remnants of evolution or key allosteric regulators? Curr. Opin. Struct. Biol. 20, 772-781.

[5] Eyers PA, Murphy JM. (2013) Exploring Kinomes: Pseudokinases and beyond: Dawn of the dead: Protein pseudokinases signal new adventures in cell biology. Biochem. Soc. Trans. 41, 969-974.

[6] Murphy JM, Zhang Q, Young SN, Reese ML, Bailey FP, Eyers PA, et al. (2014) A robust methodology to subclassify pseudokinases based on their nucleotide-binding properties. Biochem. J. 457, 323-334.

[7] Reiterer V, Eyers PA, Farhan H. (2014) Day of the dead: Pseudokinases and pseudophosphatases in physiology and disease. Trends Cell Biol. 24, 489-505.

[8] Jacobsen A V., Murphy JM. (2017) The secret life of kinases: insights into noncatalytic signalling functions from pseudokinases. Biochem. Soc. Trans. 45, 665-681.

[9] Sreelatha A, Yee SS, Lopez VA, Park BC, Kinch LN, Pilch S, et al. (2018) Protein AMPylation by an Evolutionarily Conserved Pseudokinase. Cell. 175, 809-821.

[10] Murphy JM, Farhan H, Eyers PA. (2017) Bio-Zombie: the rise of pseudoenzymes in biology. Biochem. Soc. Trans. 45, 537-544. 
1 [11] Jeffery CJ. (2019) The demise of catalysis, but new functions arise: pseudoenzymes as the phoenixes of the protein world. Biochem. Soc. Trans. 47, 371-379.

[12] Kung JE, Jura N. (2019) Prospects for pharmacological targeting of pseudokinases. Nat. Rev. Drug Discov. 18, 501-526.

[13] McMahon SB, Van Buskirk HA, Dugan KA, Copeland TD, Cole MD. (1998) The novel ATM-related protein TRRAP is an essential cofactor for the c-Myc and E2F oncoproteins. Cell. 94, 363-374.

[14] Saleh A, Schieltz D, Ting N, McMahon SB, Litchfield DW, Yates JR, et al. (1998) Tra1p is a component of the yeast Ada.Spt transcriptional regulatory complexes. J. Biol. Chem. 273, 26559-26565.

[15] Lempiäinen H, Halazonetis TD. (2009) Emerging common themes in regulation of PIKKs and PI3Ks. EMBO J. 28, 3067-73.

[16] Rivera-Calzada A, López-perrote A, Melero R, Boskovic J, Muñoz-Hernández H, Martino F, et al. (2015) Structure and Assembly of the PI3K-like Protein Kinases (PIKKs) Revealed by Electron Microscopy. AIMS Biophys. 2, 36-57.

[17] Imseng S, Aylett $\mathrm{CH}$, Maier T. (2018) Architecture and activation of phosphatidylinositol 3-kinase related kinases. Curr. Opin. Struct. Biol. 49, 177-189.

[18] Hayashi T, Hatanaka M, Nagao K, Nakaseko Y, Kanoh J, Kokubu A, et al. (2007) Rapamycin sensitivity of the Schizosaccharomyces pombe tor2 mutant and organization of two highly phosphorylated TOR complexes by specific and common subunits. Genes to cells. 12, 1357-1370.

[19] Helmlinger D, Marguerat S, Villén J, Swaney DL, Gygi SP, Bähler J, et al. (2011) Tra1 has specific regulatory roles, rather than global functions, within the SAGA co-activator complex. EMBO J. 30, 2843-2852.

[20] Manning G, Reiner DS, Lauwaet T, Dacre M, Smith A, Zhai Y, et al. (2011) The minimal kinome of Giardia lamblia illuminates early kinase evolution and unique 
parasite biology. Genome Biol. 12, R66.

[21] Díaz-Santín LM, Lukoyanova N, Aciyan E, Cheung AC. (2017) Cryo-EM structure of the SAGA and NuA4 coactivator subunit Tra1 at 3.7 angstrom resolution. Elife. 6, 120.

[22] Sharov G, Voltz K, Durand A, Kolesnikova O, Papai G, Myasnikov AG, et al. (2017) Structure of the transcription activator target Tra1 within the chromatin modifying complex SAGA. Nat. Commun. 8, 1556.

[23] Cheung ACM, Díaz-Santín LM. (2018) Share and share alike: the role of Tra1 from the SAGA and NuA4 coactivator complexes. Transcription. 00, 1-7.

[24] Sibanda BL, Chirgadze DY, Ascher DB, Blundell TL. (2017) DNA-PKcs structure suggests an allosteric mechanism modulating DNA double-strand break repair. Science. 355, 520-524.

[25] Mutiu Al, Hoke SM, Genereaux J, Liang G, Brandl CJ. (2007) The role of histone ubiquitylation and deubiquitylation in gene expression as determined by the analysis of an HTB1(K123R) Saccharomyces cerevisiae strain. Mol. Genet. Genomics. 277, 491506.

[26] Knutson BA, Hahn S. (2011) Domains of Tra1 important for activator recruitment and transcription coactivator functions of SAGA and NuA4 complexes. Mol. Cell. Biol. 31, 818-831.

[27] Berg MD, Genereaux J, Karagiannis J, Brandl CJ. (2018) The Pseudokinase Domain of Saccharomyces cerevisiae Tra1 Is Required for Nuclear Localization and Incorporation into the SAGA and NuA4 Complexes. G3 (Bethesda). 8, 1943-1957.

[28] Eyers PA, Keeshan K, Kannan N. (2017) Tribbles in the 21st Century: The Evolving Roles of Tribbles Pseudokinases in Biology and Disease. Trends Cell Biol. 27, 284298.

[29] Foulkes DM, Byrne DP, Yeung W, Shrestha S, Bailey FP, Ferries S, et al. (2018) 
Covalent inhibitors of EGFR family protein kinases induce degradation of human Tribbles 2 (TRIB2) pseudokinase in cancer cells. Sci. Signal. 11, eaat7951.

[30] Jamieson SA, Ruan Z, Burgess AE, Curry JR, McMillan HD, Brewster JL, et al. (2018) Substrate binding allosterically relieves autoinhibition of the pseudokinase TRIB1. Sci. Signal. 11, eaau0597.

[31] Park J, Kunjibettu S, McMahon SB, Cole MD. (2001) The ATM-related domain of TRRAP is required for histone acetyltransferase recruitment and Myc-dependent oncogenesis. Genes Dev. 15, 1619-1624.

[32] Bouchard C, Dittrich O, Kiermaier A, Dohmann K, Menkel A, Eilers M, et al. (2001) Regulation of cyclin D2 gene expression by the Myc/Max/Mad network: Mycdependent TRRAP recruitment and histone acetylation at the cyclin D2 promoter. Genes Dev. 15, 2042-2047.

[33] Deleu L, Shellard S, Alevizopoulos K, Amati B, Land H. (2001) Recruitment of trap required for oncogenic transformation by E1A. Oncogene. 20, 8270-8275.

[34] Lang SE, McMahon SB, Cole MD, Hearing P. (2001) E2F Transcriptional Activation Requires TRRAP and GCN5 Cofactors. J. Biol. Chem. 276, 32627-32634.

[35] Ard PG, Chatterjee C, Kunjibettu S, Adside LR, Gralinski LE, McMahon SB. (2002) Transcriptional Regulation of the mdm2 Oncogene by p53 Requires TRRAP Acetyltransferase Complexes. Mol. Cell. Biol. 22, 5650-5661.

[36] Lang SE, Hearing P. (2003) The adenovirus E1A oncoprotein recruits the cellular TRRAP/GCN5 histone acetyltransferase complex. Oncogene. 22, 2836-2841.

[37] Memedula S, Belmont AS. (2003) Sequential recruitment of HAT and SWI/SNF components to condensed chromatin by VP16. Curr. Biol. 13, 241-246.

[38] Lin L, Chamberlain L, Zhu LJ, Green MR. (2012) Analysis of Gal4-directed transcription activation using Tra1 mutants selectively defective for interaction with Gal4. Proc. Natl. Acad. Sci. U. S. A. 109, 1997-2002. 
1 [39] Brown CE, Howe L, Sousa K, Alley SC, Carrozza MJ, Tan S, et al. (2001) Recruitment of HAT complexes by direct activator interactions with the ATM-related Tra1 subunit. Science. 292, 2333-2337.

[40] Bhaumik SR, Green MR. (2001) SAGA is an essential in vivo target of the yeast acidic activator Gal4p. Genes Dev. 15, 1935-1945.

[41] Bhaumik SR, Raha T, Aiello DP, Green MR. (2004) In vivo target of a transcriptional activator revealed by fluorescence resonance energy transfer. Genes Dev. 18, 333343.

[42] Fishburn J, Mohibullah N, Hahn S. (2005) Function of a eukaryotic transcription activator during the transcription cycle. Mol. Cell. 18, 369-78.

[43] Reeves WM, Hahn S. (2005) Targets of the Gal4 Transcription Activator in Functional Transcription Complexes. Mol. Cell. Biol. 25, 9092-9102.

[44] Herbig E, Warfield L, Fish L, Fishburn J, Knutson BA, Moorefield B, et al. (2010) Mechanism of Mediator recruitment by tandem Gcn4 activation domains and three Gal11 activator-binding domains. Mol. Cell. Biol. 30, 2376-2390.

[45] Wang X, Ahmad S, Zhang Z, Côté J, Cai G. (2018) Architecture of the Saccharomyces cerevisiae NuA4/TIP60 complex. Nat. Commun. 9, 1147.

[46] Tuttle LM, Pacheco D, Warfield L, Luo J, Ranish J, Hahn S, et al. (2018) Gcn4Mediator Specificity Is Mediated by a Large and Dynamic Fuzzy Protein-Protein Complex. Cell Rep. 22, 3251-3264.

[47] Grant PA, Schieltz D, Pray-Grant MG, Yates JR, Workman JL. (1998) The ATMrelated cofactor Tra1 is a component of the purified SAGA complex. Mol. Cell. 2, 863867.

[48] Vassilev A, Yamauchi J, Kotani T, Prives C, Avantaggiati ML, Qin J, et al. (1998) The $400 \mathrm{kDa}$ subunit of the PCAF histone acetylase complex belongs to the ATM superfamily. Mol. Cell. 2, 869-875. 
1 [49] Allard S, Utley RT, Savard J, Clarke A, Grant P, Brandl CJ, et al. (1999) NuA4, an essential transcription adaptor/histone $\mathrm{H} 4$ acetyltransferase complex containing Esa1p and the ATM-related cofactor Tra1p. EMBO J. 18, 5108-19.

[50] Koutelou E, Hirsch CL, Dent SY. (2010) Multiple faces of the SAGA complex. Curr. Opin. Cell Biol. 22, 374-382.

[51] Helmlinger D, Tora L. (2017) Sharing the SAGA. Trends Biochem. Sci. 42, 850-861.

[52] Lu PY, Lévesque N, Kobor MS. (2009) NuA4 and SWR1-C: two chromatin-modifying complexes with overlapping functions and components. Biochem. Cell Biol. 87, 799815.

[53] Calonge TM, Eshaghi M, Liu J, Ronai Z, O’Connell MJ. (2010) Transformation/transcription domain-associated protein (TRRAP)-mediated regulation of Wee1. Genetics. 185, 81-93.

[54] Helmlinger D. (2012) New insights into the SAGA complex from studies of the Tra1 subunit in budding and fission yeast. Transcription. 3, 13-18.

[55] Elias-Villalobos A, Toullec D, Faux C, Lledo G, Seveno M, Helmlinger D. (2019) Chaperone-mediated ordered assembly of the SAGA transcription complex. bioRxiv.

[56] Setiaputra D, Ross JD, Lu S, Cheng DT, Dong MQ, Yip CK. (2015) Conformational flexibility and subunit arrangement of the modular yeast Spt-Ada-Gcn5 acetyltransferase complex. J. Biol. Chem. 290, 10057-10070.

[57] Han Y, Luo J, Ranish J, Hahn S. (2014) Architecture of the Saccharomyces cerevisiae SAGA transcription coactivator complex. EMBO J. 33, 2534-46.

[58] Setiaputra D, Ahmad S, Dalwadi U, Steunou A-L, Lu S, Ross JD, et al. (2018) Molecular architecture of the essential yeast histone acetyltransferase complex NuA4 redefines its multi-modularity. Mol. Cell. Biol. 38, 1-15.

[59] Mutiu AI, Hoke SMT, Genereaux J, Hannam C, MacKenzie K, Jobin-Robitaille O, et al. 
(2007) Structure/function analysis of the phosphatidylinositol-3-kinase domain of yeast Tra1. Genetics. 177, 151-166.

[60] Hoke SM, Irina Mutiu A, Genereaux J, Kvas S, Buck M, Yu M, et al. (2010) Mutational analysis of the C-terminal FATC domain of Saccharomyces cerevisiae Tra1. Curr. Genet. 56, 447-465.

[61] Takai H, Wang RC, Takai KK, Yang H, de Lange T. (2007) Tel2 Regulates the Stability of PI3K-Related Protein Kinases. Cell. 131, 1248-1259.

[62] Lustig AJ, Petes TD. (1986) Identification of yeast mutants with altered telomere structure. Proc. Natl. Acad. Sci. U. S. A. 83, 1398-402.

[63] Kanoh J, Yanagida M. (2007) Tel2: a common partner of PIK-related kinases and a link between DNA checkpoint and nutritional response? Genes to cells. 12, 13011304.

[64] Anderson CM, Korkin D, Smith DL, Makovets S, Seidel JJ, Sali A, et al. (2008) Tel2 mediates activation and localization of ATM/Tel1 kinase to a double-strand break. Genes Dev. 22, 854-859.

[65] Takai H, Xie Y, de Lange T, Pavletich NP. (2010) Tel2 structure and function in the Hsp90-dependent maturation of mTOR and ATR complexes. Genes Dev. 24, 20192030.

[66] Hurov KE, Cotta-Ramusino C, Elledge SJ. (2010) A genetic screen identifies the Triple T complex required for DNA damage signaling and ATM and ATR stability. Genes Dev. 24, 1939-1950.

[67] Kaizuka T, Hara T, Oshiro N, Kikkawa U, Yonezawa K, Takehana K, et al. (2010) Tti1 and Tel2 are critical factors in mammalian target of rapamycin complex assembly. J. Biol. Chem. 285, 20109-20116.

[68] Izumi N, Yamashita A, Hirano H, Ohno S. (2012) Heat shock protein 90 regulates phosphatidylinositol 3-kinase-related protein kinase family proteins together with the 
RUVBL1/2 and Tel2-containing co-factor complex. Cancer Sci. 103, 50-57.

[69] Ahmed S, Alpi A, Hengartner MO, Gartner A. (2001) C. elegans RAD-5/CLK-2 defines a new DNA damage checkpoint protein. Curr. Biol. 11, 1934-1944.

[70] Kim SG, Hoffman GR, Poulogiannis G, Buel GR, Jang YJ, Lee KW, et al. (2013) Metabolic stress controls mTORC1 lysosomal localization and dimerization by regulating the TTT-RUVBL1/2 complex. Mol. Cell. 49, 172-85.

[71] Shikata M, Ishikawa F, Kanoh J. (2007) Tel2 is required for activation of the Mrc1mediated replication checkpoint. J. Biol. Chem. 282, 5346-5355.

[72] Hoffman KS, Duennwald ML, Karagiannis J, Genereaux J, Alexander S, Brandl CJ. (2016) Saccharomyces cerevisiae Tti2 regulates PIKK proteins and stress response. G3 (Bethesda). 6, 1649-1659.

[73] Shevchenko AA, Roguev A, Schaft D, Buchanan L, Habermann B, Sakalar C, et al. (2008) Chromatin Central: towards the comparative proteome by accurate mapping of the yeast proteomic environment. Genome Biol. 9, R167.

[74] Inoue H, Sugimoto S, Takeshita Y, Takeuchi M, Hatanaka M, Nagao K, et al. (2017) CK2 phospho-independent assembly of the Tel2-associated stress-signaling complexes in Schizosaccharomyces pombe. Genes to Cells. 22, 59-70.

[75] Genereaux J, Kvas S, Dobransky D, Karagiannis J, Gloor GB, Brandl CJ. (2012) Genetic evidence links the ASTRA protein chaperone component Tti2 to the SAGA transcription factor Tra1. Genetics. 191, 765-780.

[76] Schopf FH, Biebl MM, Buchner J. (2017) The HSP90 chaperone machinery. Nat. Rev. Mol. Cell Biol. 18, 345-360.

[77] Verba KA, Agard DA. (2017) How Hsp90 and Cdc37 Lubricate Kinase Molecular Switches. Trends Biochem. Sci. 42, 799-811.

[78] Houry WA, Bertrand E, Coulombe B. (2018) The PAQosome, an R2TP-Based 
Chaperone for Quaternary Structure Formation. Trends Biochem. Sci. 43, 4-9.

[79] Sugimoto K. (2018) Branching the Tel2 pathway for exact fit on phosphatidylinositol 3kinase-related kinases. Curr. Genet. 64, 965-970.

[80] Goto GH, Ogi H, Biswas H, Ghosh A, Tanaka S, Sugimoto K. (2017) Two separate pathways regulate protein stability of ATM/ATR-related protein kinases Mec1 and Tel1 in budding yeast. PLoS Genet. 13, 1-22.

[81] Hammarsten O, Chu G. (1998) DNA-dependent protein kinase: DNA binding and activation in the absence of Ku. Proc. Natl. Acad. Sci. U. S. A. 95, 525-30.

[82] Aggarwal-Howarth S, Scott JD. (2017) Pseudoscaffolds and anchoring proteins: the difference is in the details. Biochem. Soc. Trans. 45, 371-379.

[83] Madeira F, Park Y mi, Lee J, Buso N, Gur T, Madhusoodanan N, et al. (2019) The EMBL-EBI search and sequence analysis tools APIs in 2019. Nucleic Acids Res. 47, W636-W641.

[84] Melero R, Uchiyama A, Castaño R, Kataoka N, Kurosawa H, Ohno S, et al. (2014) Structures of SMG1-UPFs complexes: SMG1 contributes to regulate UPF2-dependent activation of UPF1 in NMD. Structure. 22, 1105-1119.

[85] Yang H, Jiang X, Li B, Yang HJ, Miller M, Yang A, et al. (2017) Mechanisms of mTORC1 activation by RHEB and inhibition by PRAS40. Nature. 552, 368-373.

[86] Chen X, Liu M, Tian Y, Li J, Qi Y, Zhao D, et al. (2018) Cryo-EM structure of human mTOR complex 2. Cell Res. 28, 518-528.

[87] Baretić D, Pollard HK, Fisher DI, Johnson CM, Santhanam B, Truman CM, et al. (2017) Structures of closed and open conformations of dimeric human ATM. Sci. Adv. 3, e1700933.

[88] Wang X, Ran T, Zhang X, Xin J, Zhang Z, Wu T, et al. (2017) $3.9 \AA$ structure of the yeast Mec1-Ddc2 complex, a homolog of human ATR-ATRIP. Science. 358, $1206-$ 
1209.

2 [89] Rose AS, Bradley AR, Valasatava Y, Duarte JM, Prlic A, Rose PW. (2018) NGL

3 viewer: Web-based molecular graphics for large complexes. Bioinformatics. 34, 3755-

4 3758.

5

6 
HEAT

FAT

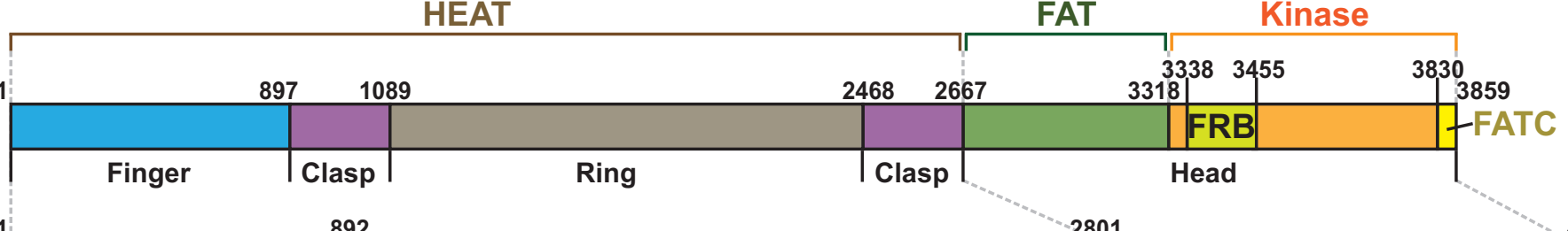

1

892

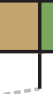

Head

$\mathrm{N}$-terminal unit

Circular Cradle unit

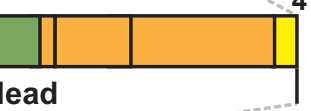

4128

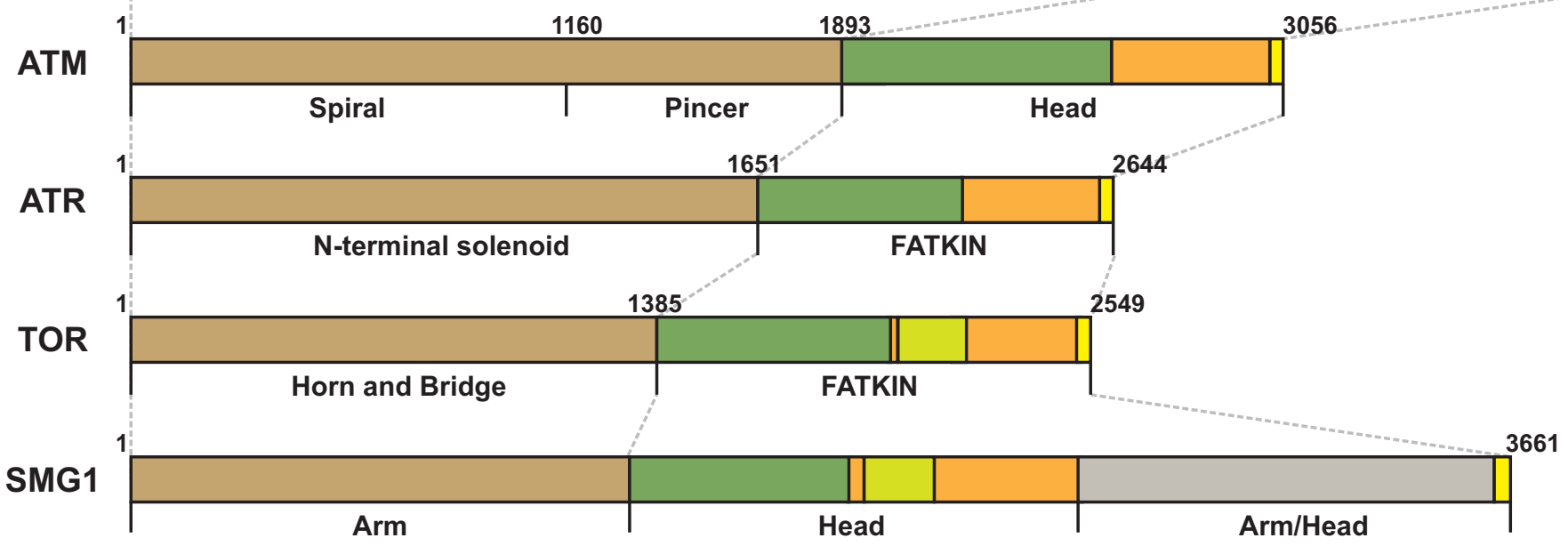

ATR GSDGKFYI MMCK PKDDI -2332

ATM GSDGKERROLVKGRDDL -2722

TOR GSNGHEFVFLLKGHEDL -2192

SMG1 GSDGKSYPYLFKGLEDL -2160

DNA-PK GHDEREHPFIVKGGEDI -3758

TRRAP GHNGK I Y PY LVMNDACL -3536

PI3Kc TEDGGKY PVI FRH GDDI -641

$$
\begin{gathered}
\text { VAIK } \\
\text { (ATP binding) }
\end{gathered}
$$

SpTra2 GYDGKLYPFALQY PATR -3321 SpTra1 SNGGTIHPFVIOY P SAR -3368 ScTra1 GHDGSVH SFAV Q Y PAVR -3411 NcTra1 GHDGSVH SFAVOH PAAR -3572 AtTra1 GSDGSQKHEIVOT S I T P -3522 HsTRRAP GHNGKIY PY LVMNDACL -3536 MmTrrap GHNGK I Y PY LVMND ACL -3524 DrTrrap GHNGKI Y PY LVMNDACL -3500 DmTrrap GTNGKIY PYLVVILD SGL -3478
RS AYCRSTAVMSMVGY I LGLGDRHGEN I IFD S LTGE CVAVDFNC LFNKGE -2503 RLAYTRSVAT SS IVGY I LGLGDRHVQN I I INE $Q S A E L V H$ IDLGVAFE $Q$ GK -2898 RTNYTRSIAVMSMVGY I LGLGDRHP SNLMLDRL SGK I LH IDFGD CFEVAM-2366 TQSYARSTAVMSMVGY I IGLGDRHLDNVII DMTTGEVVH IDYNVCFEKGK-2363 RS HFASSHAL I C I SHWI L GIGDRHLNNFMVAMETGGV I GID FGHAFG SA T -3919 RKMFT I QLALI GFAE FVLHLNRLN PEMLQ I A QDTGK LNVAYFRFD INDAT -3718 MD TYVKSCAAG Y V I TY I L GV GDRHLDNILILT - KT GK L FH IDFG I L GRD P -771

\footnotetext{
DXXXXN

(catalytic loop)

(Mg ${ }^{2+}$ binding)
}

RRTFTTQYAYMI IMTYVFNIGGRSPRLFIVKDSGQV- - -I-MS'QDL -3514 RKNFSYQYAC F SEITYIIS INNRI PAKLVFSRD S GGV- - --WTITEA -3557 RKQFAS QY S S FVFMS YMMMI NNRT PHK I HVDKT S GNV - - - FT'LEM -3586 RRRF S Y Q LAAL T FMT Y I IH IDIKRY PHKFNIAR GS SN I - - - - W WG E L -3753 KKQFAVQLAVS S FMS FML Q I GGRS PNKVI FAKNTGKM- - -I-FO'TDF -3697 RKMFTI QLAL I GFAE FVI H LNIR LNPEMIQI AQDT GKLNVA|Y FR'FD I -3714 RKMFT I QLAL I GFAE FVI LNR LN PEMI I AQDT TKLNVAY FRIFD I -3702 RKMF T I Q LA L I GLAE F ML H LNR L N P E MLO I AQDT GKL NV SYY FRFD I -3678 RKMLTLQLALA F L CE HALN L T R L NADMMY L H QD S GLMN I S|YF K F DV -3656 
A

\section{Amorphea}

Metazoa

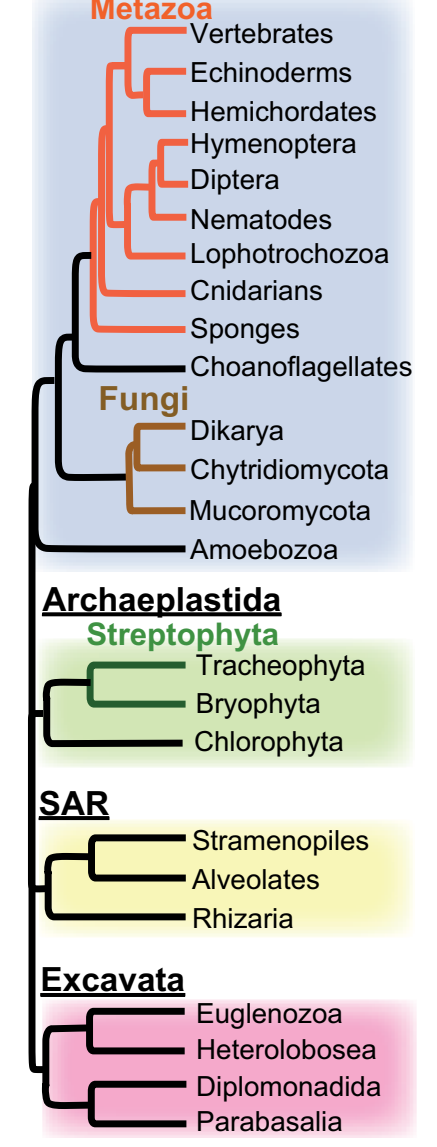

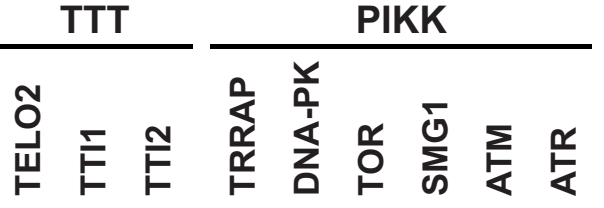

$\begin{aligned} & - \\ & -\end{aligned}-\begin{aligned} & \text { H. sapiens, G. gallus, D. rerio } \\ & \text { S. purpuratus }\end{aligned}$

S. kowalevskii

$\bigcirc \bigcirc \bigcirc$ A. echinatior

\

- $\square$

Х

- D. melanogaster, B. oleae, A. aegypti

- C. elegans, W. bancrofti, B. malayi

C. gigas

- $H$. vulgaris, N. vectensis, A. digitifera

- A. queenslandica

M. brevicollis

$\begin{array}{ll}0 & 0 \\ 0 & 0 \\ 0 & 0\end{array}$

a $\triangle 00$ ○

- S. cerevisiae, S. pombe, A. bisporus

- S. punctatus, $N$. californiae

- B. adelaidae

D. discoideum b $\bigcirc$ S. moelledorffii

- $\bigcirc \bigcirc$ P.patens

$\bigcirc \quad \square \quad$ C. reinhardtii

B

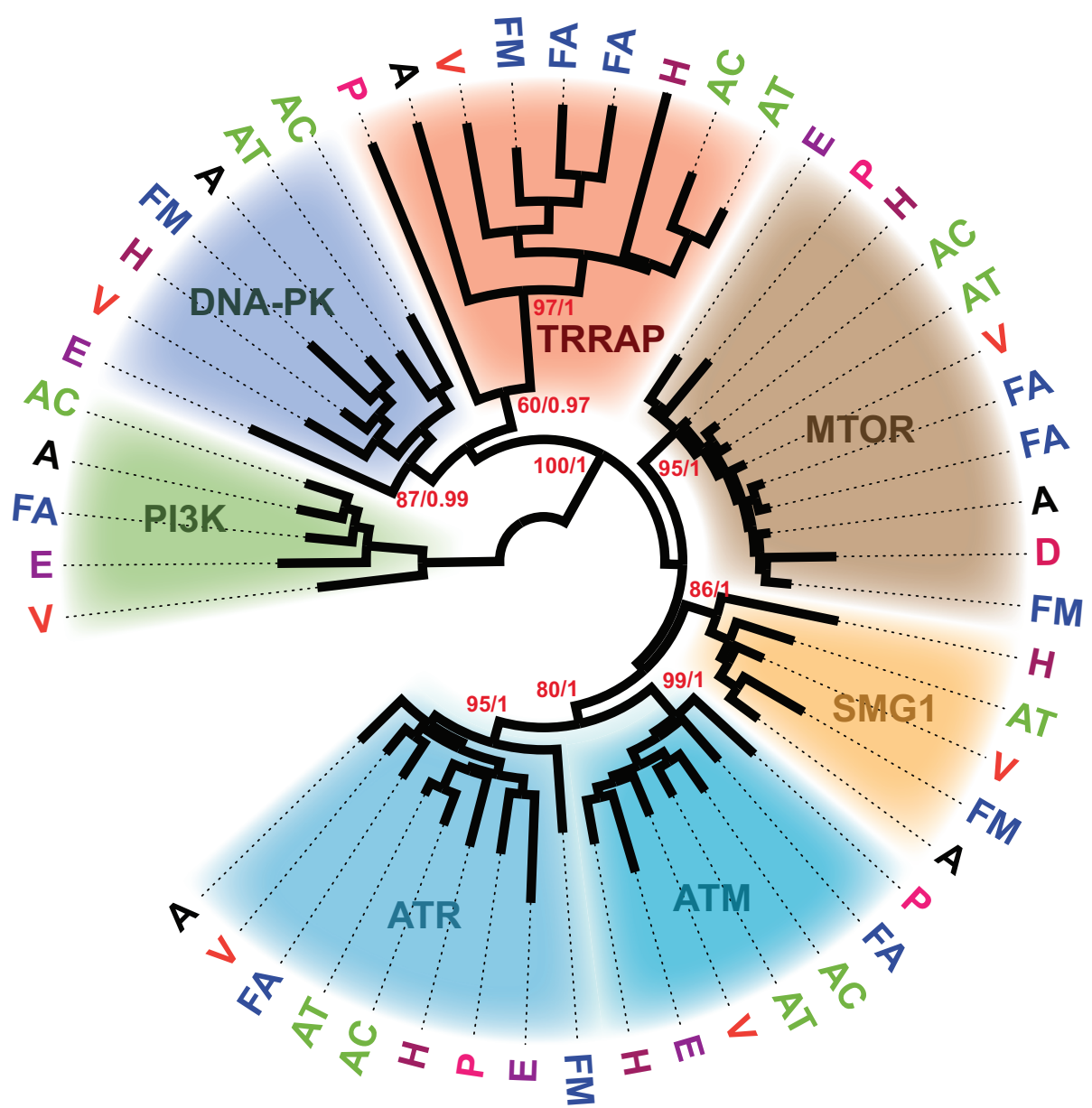




\section{SMG1 complex} (SMG1/8/9-UPF1/2)
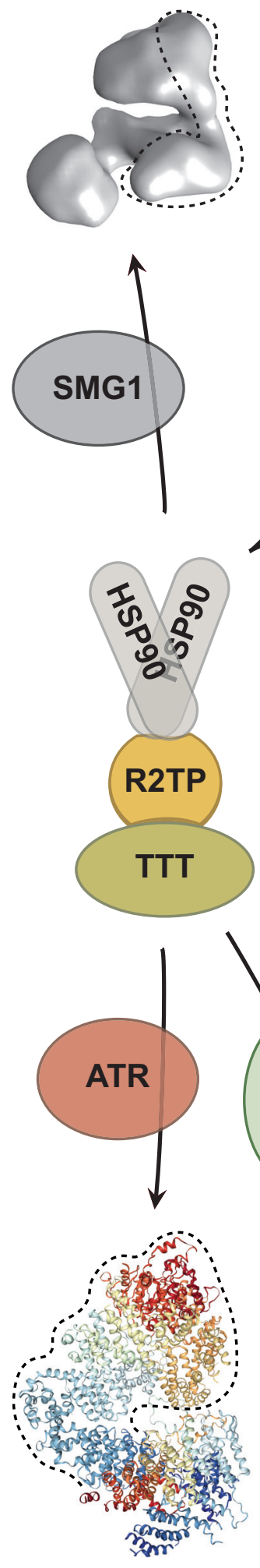

ATR
mTORC1 complex

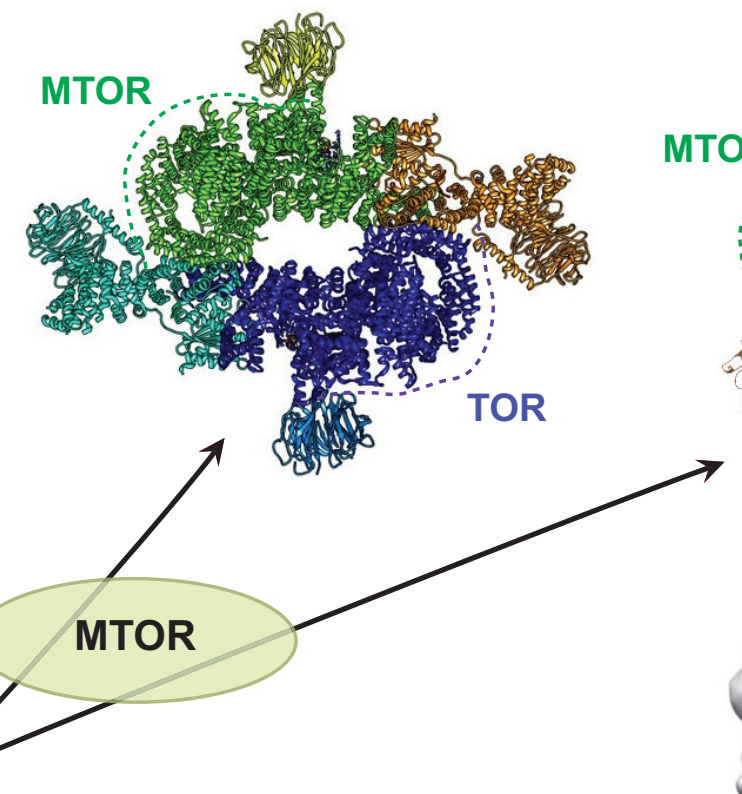

mTORC2 complex

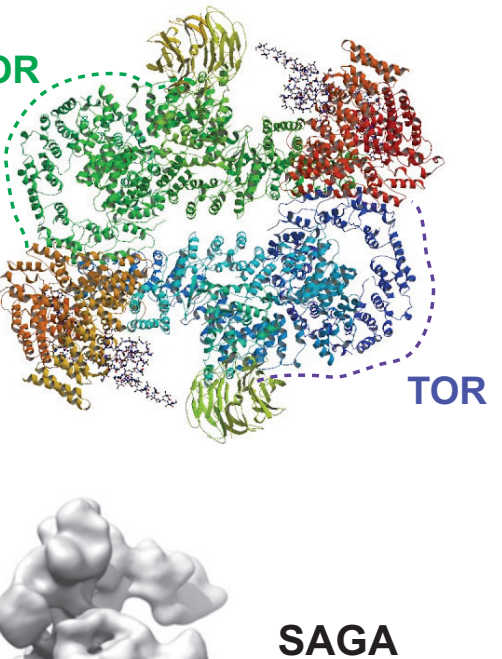

complex

(Mec1-Ddc2 complex)

NuA4

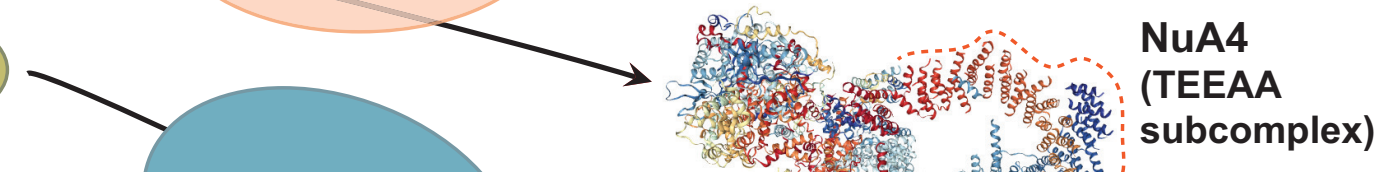

TRRAP

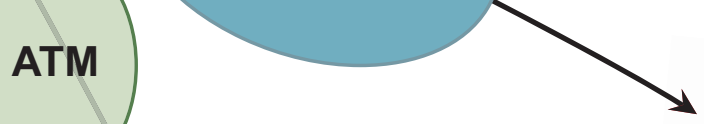

TRRAP
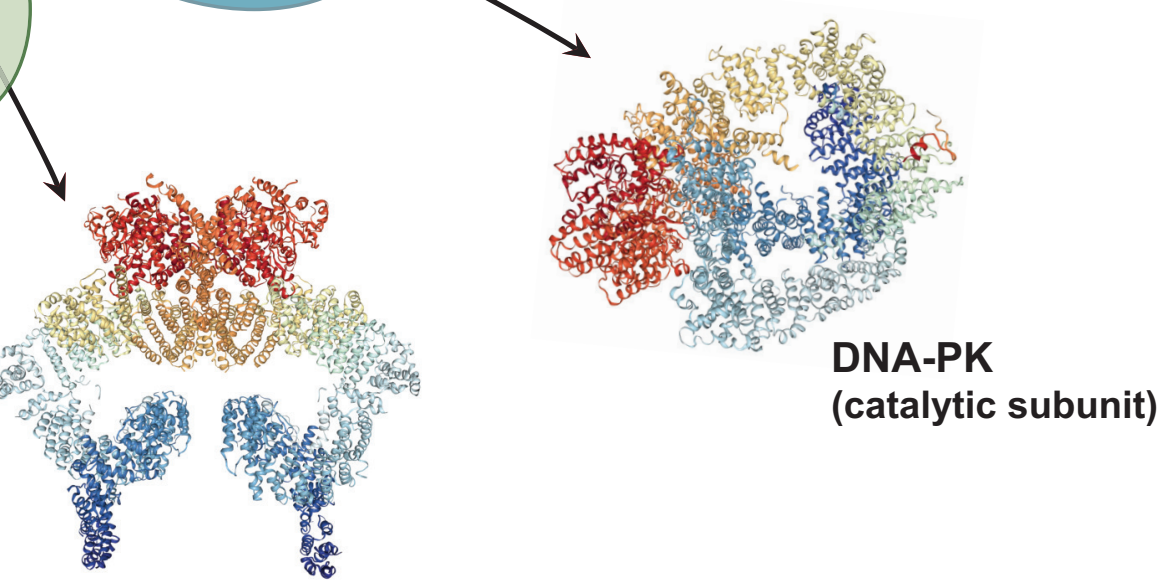

(catalytic subunit)

\section{ATM}

(closed dimer) 